\title{
Tecnologia móvel e ubíqua na educação: uma análise com base em revisão sistemática.
}

\author{
Gabriel de Oliveira Araújo ${ }^{1}$, Gabriel de Oliveira Ferreira ${ }^{1}$, Danielle Pompeu \\ Noronha ${ }^{1}$.
}

${ }^{1}$ Escola Superior de Tecnologia - Universidade do Estado do Amazonas - UEA - Brasil Av. Darcy Vargas CEP 80215-901 - Manaus - AM

${ }^{1}$ Licenciando em Informática, Coordenação de Licenciatura em Informática ${ }^{2}$

\{goa.inf, gof.inf\}@uea.edu.br, danipnoronha@gmail.com

Resumo: As tecnologias móveis e ubíquas estão se tornando tendências cada vez mais utilizadas no processo educacional e é notória a necessidade de um estudo aprofundado em relação ao tema. O seguinte artigo tem como objetivo analisar a relevância dos materiais científicos, metodologias e resultados mensurados nos estudos dessas tendências no processo de ensino/aprendizagem, usando a revisão sistemática como metodologia para verificar se as mesmas são favoráveis à educação. O protocolo é composto por quatro questões de pesquisa, dentre elas destaca-se a quarta "Quais as contribuições positivas foram viabilizadas pelas tecnologias móveis e ubíquas em sistemas para ensino/aprendizagem?". A aplicação dos critérios de exclusão e inclusão culminou na seleção e análise de 19 artigos publicados no Simpósio Brasileiro de Informática na Educação - SBIE entre 2007 e 2017, além do uso de uma string de busca relacionada ao tema. Dentre os resultados obtidos destaca-se a contribuição positiva destas tecnologias, como exemplo o aumento de engajamento, o incentivo e a concentração no processo de ensino/aprendizagem.

\begin{abstract}
Mobile and ubiquitous technologies are becoming increasingly used trends in the educational process and the need for in-depth study on the subject is evident. The following article aims to analyze the relevance of scientific materials, methodologies and results measured in the study of these trends in the teaching / learning process, using the systematic review as a methodology to verify if they are favorable to education. The protocol is composed of four research questions, among them the fourth one: "What positive contributions have been made possible by mobile and ubiquitous technologies in teaching / learning systems?" The application of exclusion and inclusion criteria culminated in the selection and analysis of 19 articles published in the Brazilian Symposium on Informatics in Education (SBIE) between 2007 and 2017, in addition to the use of a search string related to the topic. Among the results obtained, we highlight the positive contribution of these technologies, such as increased engagement, encouragement and concentration in the teaching / learning process.
\end{abstract}


VI Congresso Brasileiro de Informática na Educação (CBIE 2017)

Anais do XXIII Workshop de Informática na Escola (WIE 2017)

\section{Introdução}

O surgimento das tecnologias móveis e ubíquas desencadeou uma série de investigações sobre a possibilidade de aplicação das mesmas no âmbito educacional. Diante desse contexto, o objetivo desse trabalho foi o desenvolvimento de uma Revisão Sistemática de Literatura - RSL - com a finalidade de averiguar a contribuições das diferentes metodologias, aplicações e resultados encontrados nos processos de ensino/aprendizagem utilizando tecnologias móveis e ubíquas como ferramenta.

A RSL foi utilizada visando levantar, reunir, catalogar, analisar e descrever a relevância das publicações que remetem ao tema, extraindo as contribuições para o ensino/aprendizagem. Além desta seção de introdução, o artigo está estruturado da seguinte forma: a seção 2 apresenta o referencial teórico que embasa a RSL, a seção 3 descreve a metodologia usada, a seção 4 discute a análise dos resultados e a seção 5 apresenta as considerações finais e os trabalhos futuros.

\section{Referencial Teórico}

Com o avanço da tecnologia a escola deve estar atenta para as interferências e mudanças que ocorrem no cotidiano dos alunos, por isso deve pensar na tecnologia como auxílio no processo de ensino e aprendizagem dos discentes, de modo a contribuir na construção do conhecimento.

Moran (2012) defende que não se deve separar a realidade dos alunos do contexto escolar, pois uma escola que não está conectada não está completa. A realidade das escolas do século XX consiste em usar recursos tecnológicos para dinamizar e representar os conteúdos vistos em sala de aula. Entre os recursos disponíveis têm-se softwares, jogos, notebooks e os dispositivos móveis. Os dispositivos móveis permitem, num sentido literal, usar recursos tecnológicos em sala de aula, assim como promover a inclusão de ferramentas que estão ao alcance dos alunos e professores, e que muitas vezes têm utilidade não educativa.

Como alternativa para a utilização de tecnologias móveis na educação Mobile Learning vem sendo amplamente difundida como concepção de novos modelos pedagógicos que usam dispositivos móveis, como celulares, tablets, PDA, etc na sala de aula (Ally \& Prieto-Blázquez, 2014; Cobcroft, Towers, Smith, \& Bruns, 2006; Traxler, 2005; Wu et al., 2012).

Pelissoli e Loyolla (2004) define o M-Learning como a fusão de tecnologias de processamento e comunicação de dados com a finalidade de proporcionar uma maior interação com grupo de estudantes e aos professores. Portanto, o M-Learning une tecnologias de redes sem fio na aprendizagem. Ainda segundo o autor para utilizar os dispositivos móveis é importante que o professor trace estratégias que sincronizem o uso da ferramenta com seus conteúdos didáticos, entre elas há: O smartphone, na qual os alunos podem receber mensagens com questões referentes ao assunto; utilizar grupos de whatsapp para interagir com os conteúdos; acessar jogos digitais com temática da aula, etc. O Tablet, elaborar criações de histórias animadas, dependendo dos softwares instalados, realizar pesquisas para os assuntos da aula, analisar imagens enviados pelo professor a respeito do conteúdo.

Acredita-se que, o uso correto de ações baseadas no m-learning facilita a comunicação entre alunos e professores no processo de ensino-aprendizagem, de modo a trazer para sala de aula o mundo real e confirmando que é possível aprender através de diversos recursos. 
VI Congresso Brasileiro de Informática na Educação (CBIE 2017)

Anais do XXIII Workshop de Informática na Escola (WIE 2017)

\section{Metodologia}

Essa revisão sistemática é estruturada para o levantamento de materiais científicos publicados entre os anos de 2007 e 2017, procurando analisar as principais aplicações e resultados verificados usando tecnologias móveis e ubíquas na educação, bem como considerar panoramicamente as pesquisas na área, procurando levantar questões pertinentes às novas tendências. Fizeram parte desta RSL artigos de 01 de janeiro de 2007 a 28 de fevereiro de 2017.

\subsection{Processo de busca, critérios de inclusão/exclusão e string de busca}

O escopo de mapeamento constituiu-se de 3 etapas: 1) utilizar o motor de busca do Simpósio Brasileiro de Informática na Educação (SBIE); 2) definir filtro para mapeamento, como: objetivos, questões de pesquisa, critérios de exclusão, critérios de inclusão e string de busca; e, 3) realizar buscas manuais no motor de busca definido.

Iniciou-se o mapeamento e construção do banco de artigos, procurando responder questões apontadas (ver Tabela 1).

Tabela 1: Questões de Pesquisa

\begin{tabular}{|c|l|}
\hline IDENTIFICADOR & \multicolumn{1}{|c|}{ QUESTÕES DE PESQUISA } \\
\hline QP1 & $\begin{array}{l}\text { As tecnologias móveis e ubíquas são de fácil acesso e } \\
\text { gratuitas? }\end{array}$ \\
\hline QP2 & $\begin{array}{l}\text { Quais os níveis de escolaridade estão sendo submetidos a } \\
\text { esses tipos de tecnologias? }\end{array}$ \\
\hline QP3 & $\begin{array}{l}\text { Os resultados da aplicação das tecnologias móveis e ubíquas } \\
\text { são favoráveis ao ensino/aprendizagem? }\end{array}$ \\
\hline QP4 & $\begin{array}{l}\text { Quais as contribuições positivas foram viabilizadas pelas } \\
\text { tecnologias móveis e ubíquas em sistemas para } \\
\text { ensino/aprendizagem? }\end{array}$ \\
\hline
\end{tabular}

Realizou-se em quatro etapas o processo de busca. Na primeira etapa, foram recuperadas todas as publicações disponíveis no site oficial do Simpósio Brasileiro de Informática na Educação. Para o SBIE, todas as publicações entre 01 de janeiro de 2007 a 28 de fevereiro de 2017 foram recuperados 522 estudos primários, que posteriormente foram analisados a fim de verificar a relevância para responder as questões de pesquisa. Na segunda etapa, aplicou-se os Critérios de Exclusão (CE) com o objetivo de preterir publicações que se encaixam nos critérios expostos (ver Tabela 2). A aplicação dos Critérios de Exclusão culminou obviamente na exclusão de trabalhos que não atendessem pelo menos um dos critérios. Após realizado a leitura dos títulos, palavras chaves, resumos, este conjunto inicial reduziu-se para 37.

Tabela 2: Critérios de Exclusão

\begin{tabular}{|c|l|}
\hline CRITÉRIO & \multicolumn{1}{|c|}{ DEFINIÇÃO DOS CRITÉRIOS DE EXCLUSÃO } \\
\hline$C E 1$ & E1 - Estudos que não se refiram às tecnologias móveis e ubíquas. \\
\hline$C E 2$ & $\begin{array}{l}\text { E2 - Estudos que estejam em idiomas diferentes do português, } \\
\text { dificultando a coleta e a identificação de informações; }\end{array}$ \\
\hline
\end{tabular}


VI Congresso Brasileiro de Informática na Educação (CBIE 2017)

Anais do XXIII Workshop de Informática na Escola (WIE 2017)

\begin{tabular}{|l|l|}
\hline CE3 & $\begin{array}{l}\text { E3 - Estudos que não estejam disponíveis para download na base de } \\
\text { dados. }\end{array}$ \\
CE4 & $\begin{array}{l}\text { E4 - Artigos publicados antes de 01 de janeiro de 2007 e depois de } \\
28 \text { de fevereiro de 2017. }\end{array}$ \\
\hline
\end{tabular}

A terceira etapa procedeu com a leitura dos resumos e considerações finais, selecionando então 25 trabalhos. Nessa etapa, aplicou-se os Critérios de Inclusão (CI) (ver Tabela 3), procurando identificar o enfoque das publicações selecionadas.

Tabela 3: Critérios de Inclusão

\begin{tabular}{|l|l|}
\hline \multicolumn{1}{|c|}{ CRITÉRIO } & \multicolumn{1}{|c|}{ DEFINIÇÃO DOS CRITÉRIOS DE INCLUSÃO } \\
\hline CI1 & $\begin{array}{l}\text { Estudos em relação ao desenvolvimento de tecnologias móveis e } \\
\text { ubíquas para ensino/aprendizagem. }\end{array}$ \\
\hline CI2 & $\begin{array}{l}\text { Estudos que contemplam a análises da aprendizagem via tecnologia } \\
\text { móvel ou ubíqua. } \\
\text { Estudos de contribuições viabilizadas pelas tecnologias móvel e } \\
\text { ubíqua em sistemas para ensino/aprendizagem. }\end{array}$ \\
\hline CI3 & \begin{tabular}{l} 
Estudos que possibilitam trabalhos futuros \\
\hline
\end{tabular} \\
\hline
\end{tabular}
inclusão.

Todos os trabalhos selecionados cumpriram pelo menos um dos critérios de

A quarta etapa seguiu com a leitura por completo, objetivando somente 19 dos trabalhos para compor a seleção final.

A busca seletiva para realização de RSL foi concretizada usando as strings de busca (ver Tabela 40.

Tabela 4: Strings de Busca Utilizadas

\begin{tabular}{|c|c|}
\hline IDIOMA & STRING DE BUSCA \\
\hline Português & (“móvel”) ou (“aprendizagem móvel”) ou (“ubiqua”). \\
\hline Inglês & ("mobile”) or (Mobile learning, m-learning) or (“ubiquitos"). \\
\hline Espanhol & ("movil”) or (“Aprendizaje móvil)" or ("ubicuo"). \\
\hline
\end{tabular}

\subsection{Extração dos dados}

$\mathrm{Na}$ fase de extração de dados sobre os estudos selecionados, foram extraídos seguintes: objetivos do estudo; método do estudo; de que forma os dados foram coletados e considerados durante o estudo; escolaridade em que foi aplicado a pesquisa; tipos de tecnologias móveis e ubíquas que foram usadas; as metodologias de aplicação; as dificuldades encontradas na aplicação; os resultados e considerações; houve benefícios do uso das tecnologias móveis e ubíquas no ensino/aprendizagem. 
VI Congresso Brasileiro de Informática na Educação (CBIE 2017)

Anais do XXIII Workshop de Informática na Escola (WIE 2017)

\subsection{Análise e síntese}

O resultado de busca foi baseado em títulos, resumos e também palavras chave, de um total de 522 publicações, somente 37 foram pré-selecionadas após a aplicação dos Critérios de Exclusão. Posteriormente, foram analisados todos os resumos e as conclusões desses 37 estudos e aplicados os Critérios de Inclusão. Após essa segunda análise, 18 artigos foram excluídos, sendo relevante um restante de 19 selecionados para a fase de extração de dados, como mostra a Tabela 5. Os artigos foram classificados em quatro tópicos, segundo os objetivos propostos.

Tabela 5: Trabalhos finais

\begin{tabular}{|c|c|c|c|c|}
\hline Q. & Título & Ano & Autor(es) & Classificação \\
\hline 1 & $\begin{array}{l}\text { Um Modelo de Adaptação de } \\
\text { Objetos de Aprendizagem com } \\
\text { foco em Dispositivos Móveis }\end{array}$ & 2012 & $\begin{array}{c}\text { Márcia A., Cristiano A., } \\
\text { Jorge B., Sandro R., } \\
\text { Wagner C. }\end{array}$ & \multirow{10}{*}{$\begin{array}{c}\text { Mostrar o projeto e/ou } \\
\text { construção de aplicações } \\
\text { com fins educacionais para } \\
\text { dispositivos móveis. }\end{array}$} \\
\hline 2 & $\begin{array}{l}\text { Projeto e Desenvolvimento de } \\
\text { um Aplicativo de Geometria } \\
\text { Interativa para Dispositivos } \\
\text { Móveis }\end{array}$ & 2008 & $\begin{array}{c}\text { Laís Z, Simone S., } \\
\text { Aparecida M., João P., } \\
\text { Anarosa A., Leônidas O., } \\
\text { Seiji I. }\end{array}$ & \\
\hline 3 & $\begin{array}{l}\text { Modelagem de um Framework } \\
\text { para M-Learning }\end{array}$ & 2010 & $\begin{array}{c}\text { Sandra D., Érico M., } \\
\text { Solange L., Roseclea D. }\end{array}$ & \\
\hline 4 & $\begin{array}{l}\text { MOBILE HQ: O USO DE } \\
\text { SOFTWARES EDUCATIVOS } \\
\text { NA MODALIDADE M- } \\
\text { LEARNING }\end{array}$ & 2012 & $\begin{array}{l}\text { Andreia R., Patricia M., } \\
\text { Roseclea } \\
\text { D., Leander C., Rodrigo } \\
\text { C., Rodrigo L. }\end{array}$ & \\
\hline 5 & $\begin{array}{c}\text { Geomóvel: Um Aplicativo para } \\
\text { Auxílio a Aulas de Campo de } \\
\text { Geologia }\end{array}$ & 2013 & $\begin{array}{l}\text { Edgar M., Rossana M., } \\
\text { Windson V., Daniel R., } \\
\text { Márcio M., Wendell M., } \\
\text { Jamile F., Witalo B. }\end{array}$ & \\
\hline 6 & $\begin{array}{l}\text { SIGAA Mobile - O caso de } \\
\text { sucesso da ferramenta de gestão } \\
\text { acadêmica na era da } \\
\text { computação móvel }\end{array}$ & 2013 & $\begin{array}{c}\text { Itamir B., Gibeon A., José } \\
\text { G. }\end{array}$ & \\
\hline 7 & $\begin{array}{c}\text { Especificação e } \\
\text { desenvolvimento de um } \\
\text { ambiente educativo móvel para } \\
\text { a prática da escrita Braille }\end{array}$ & 2014 & $\begin{array}{c}\text { Antonio R., Agebson R., } \\
\text { Windson V., José A., } \\
\text { Jaime S. }\end{array}$ & \\
\hline 8 & $\begin{array}{c}\text { Desenvolvimento de um } \\
\text { aplicativo móvel educacional } \\
\text { voltado ao ensino de Estrutura } \\
\text { de dados }\end{array}$ & 2016 & $\begin{array}{c}\text { Luiz R., Ian S, Diego F., } \\
\text { Aneil M. }\end{array}$ & \\
\hline 9 & $\begin{array}{l}\text { Ortomonstro: um audiogame } \\
\text { móvel customizável para } \\
\text { práticas ortográficas de } \\
\text { português por meio do Braille }\end{array}$ & 2016 & $\begin{array}{c}\text { Maria C., Bruno R., } \\
\text { Jonathan G. e } \\
\text { Windson V. }\end{array}$ & \\
\hline 10 & $\begin{array}{l}\text { Teoria Fundamentada nos dados } \\
\text { para o projeto da mecânica de } \\
\text { jogo educacional móvel para } \\
\text { adultos mais velhos }\end{array}$ & 2016 & Ronan L., Lucila I. & \\
\hline 11 & $\begin{array}{l}\text { Estudo e Definição de um } \\
\text { Conjunto de Características e } \\
\text { Requisitos para Ambientes de }\end{array}$ & 2012 & Nemésio F., Ellen F. & $\begin{array}{l}\text { Analisar e/ou relatar } \\
\text { resultados a partir do uso }\end{array}$ \\
\hline
\end{tabular}


VI Congresso Brasileiro de Informática na Educação (CBIE 2017)

Anais do XXIII Workshop de Informática na Escola (WIE 2017)

\begin{tabular}{|c|c|c|c|c|}
\hline & Aprendizagem Móvel & & & \multirow{5}{*}{$\begin{array}{c}\text { das tecnologias móveis e } \\
\text { ubíquas }\end{array}$} \\
\hline 12 & $\begin{array}{c}\text { Benefícios da Computação } \\
\text { Pervasiva na Educação e } \\
\text { mobUS,Um Sistema Móvel no } \\
\text { Auxilio à Aprendizagem }\end{array}$ & 2012 & Ricardo B., Diego P. & \\
\hline 13 & $\begin{array}{l}\text { EduConnect: uma ferramenta de } \\
\text { apoio à aprendizagem } \\
\text { colaborativa para dispositivos } \\
\text { móveis em redes MANET }\end{array}$ & 2013 & José W., Luís C. & \\
\hline 14 & $\begin{array}{l}\text { Validação de um Ambiente de } \\
\text { Aprendizagem Móvel em Curso } \\
\text { a Distância }\end{array}$ & 2009 & $\begin{array}{c}\text { Patric S., Fábio T., Patrícia } \\
\text { M., Roseclea D. }\end{array}$ & \\
\hline 15 & $\begin{array}{l}\text { M-learnMat: Aplicação de um } \\
\text { Modelo Pedagógico para } \\
\text { Atividades de M-learning em } \\
\text { Matemática }\end{array}$ & 2011 & $\begin{array}{l}\text { Silvia C., Patricia A., } \\
\text { Liliana M. }\end{array}$ & \\
\hline 16 & $\begin{array}{c}\text { Ambientes Virtuais de } \\
\text { Aprendizagem e Ambientes } \\
\text { Imersivos: um estudo de caso } \\
\text { utilizando tecnologias de } \\
\text { computação móvel }\end{array}$ & 2013 & $\begin{array}{l}\text { Gleizer B., Felipe B., } \\
\text { Fabricio H., Roseclea D. }\end{array}$ & \multirow{2}{*}{$\begin{array}{l}\text { Integração de material } \\
\text { disponível em AVAs com } \\
\text { smartphones }\end{array}$} \\
\hline 17 & $\begin{array}{c}\text { M-AVA: Modelo de } \\
\text { Adaptabilidade para Ambientes } \\
\text { Virtuais Móveis de } \\
\text { Aprendizagem }\end{array}$ & 2016 & $\begin{array}{c}\text { Viviane F, Marília A., } \\
\text { Maria I. }\end{array}$ & \\
\hline 18 & $\begin{array}{l}\text { Um Modelo para Colaboração } \\
\text { em Ambientes Descentralizados } \\
\text { de Educação Ubíqua }\end{array}$ & 2012 & $\begin{array}{l}\text { Solon R., Jorge L., Jezer } \\
\text { O., André W., Débora N., } \\
\text { Patrícia B. }\end{array}$ & \multirow{2}{*}{ Outros } \\
\hline 19 & $\begin{array}{c}\text { mAbES: Avaliação de uso de } \\
\text { uma aplicação móvel para } \\
\text { usuários que são cegos }\end{array}$ & 2015 & $\begin{array}{l}\text { Marcia B., Juliana D., } \\
\text { Tasmay I. }\end{array}$ & \\
\hline
\end{tabular}

\section{Resultados e discussões}

Após a análise de todos os trabalhos finais, realizou-se a organização dos mesmo por ano de publicação com o objetivo de verificar qual o panorama e popularidade de pesquisas relacionado ao tema nos últimos 10 anos, como mostra o Gráfico 1.

\section{Gráfico 1: Quantidade de trabalhos organizados por ano de publicação}

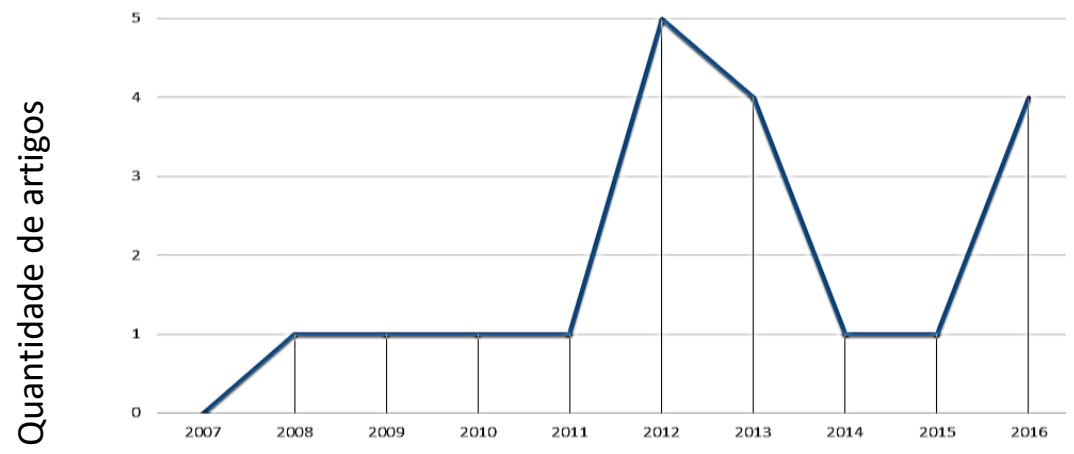

Ano 
Respondendo a primeira questão de pesquisa "As tecnologias móveis e ubíquas são de fácil acesso e gratuitas? ", o uso das tecnologias móveis e ubíquas é indispensável no dia a dia, e por essa razão são consideradas acessíveis e de fácil manuseio.

Pode-se observar a popularidade dos dispositivos com sistema Android, tais como os da linha Samsung Galaxy, por exemplo. O gráfico a seguir mostra a divisão em porcentagem do sistema operacional mais utilizados nas pesquisas.

\section{Gráfico 2: Sistemas operacionais mais usados nos trabalhos finais}

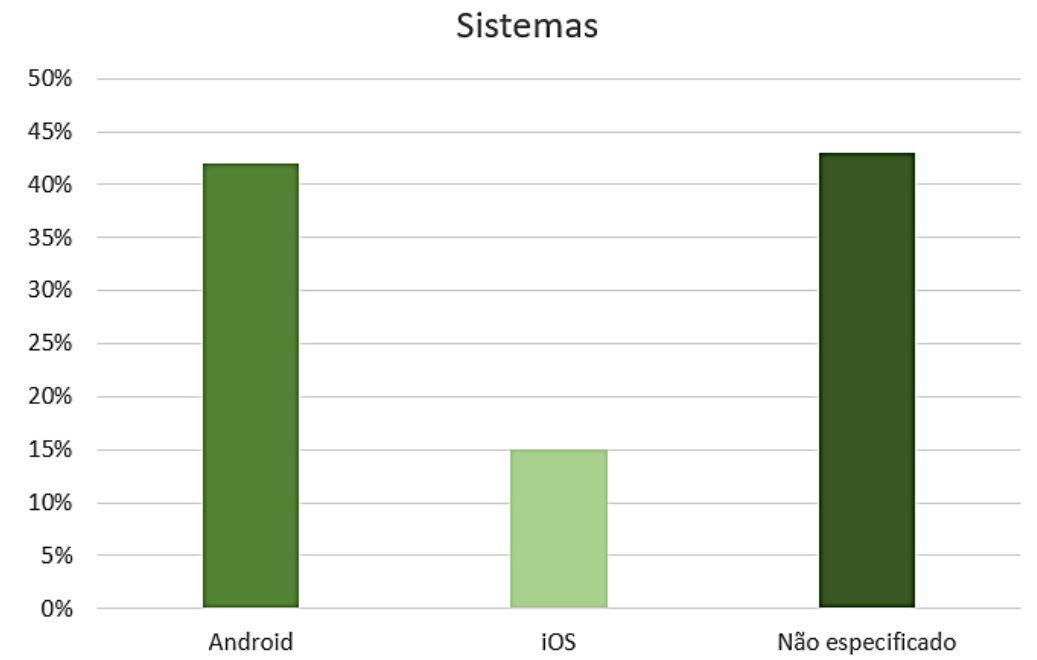

Dispositivos das marcas Nokia, Motorola, LG, iPhone, e tablets Samsung e Apple foram usados para aplicação dos testes. Cerca de $43 \%$ dos trabalhos não especificaram qual tipo de dispositivos foram usados. As principais funcionalidades dos dispositivos utilizados nas pesquisas são câmeras para registros, blocos de notas para anotações, navegadores (browsers) para acesso a material online e conexões via bluetooth e wi-fi direct para compartilhamento de documentos.

Considerando a segunda questão de pesquisa "Quais os níveis de escolaridade estão sendo submetidos a esses tipos de tecnologias? ", cerca de 47\% dos trabalhos não especificaram o público a qual se dirigiu o estudo ou aplicação das metodologias, $22 \%$ foi direcionado a um público que inclui ensino fundamental e idosos. Um percentual de aproximadamente $31 \%$ dos estudos foram direcionados ao ensino superior.

Verificou-se que atualmente várias aplicações estão sendo desenvolvidas e implementadas na m-learning, cerca de 52,6\% dos estudos pretendiam mostrar o projeto e/ou construção dessas aplicações com fins educacionais para dispositivos móveis. Cerca de $26,4 \%$ dos estudos dedicaram-se a analisar e relatar resultados do uso das tecnologias móveis nos sistemas de ensino aprendizagem e 10,5\% propuseram a integração Ambientes Virtuais de Aprendizagem - AVAs com dispositivos móveis. Os outros 10,5\% tinha objetivos diferentes desses apontados. Em alguns casos, foram encontras dificuldades na aplicação das metodologias propostas pelas pesquisas, sendo as mais comuns a falta de acesso à internet de qualidade e a disposição do conteúdo em dispositivos de tamanho de tela reduzido que dificultaram a visualização de imagens e letras, o que não comprometeu a pesquisa completamente.

Em relação à terceira questão de pesquisa "Os resultados da aplicação das tecnologias móveis e ubíquas são favoráveis ao ensino/aprendizagem? ", constatou-se que os resultados dos estudos que foram analisados (muito deles produtos de aplicações 
de testes e questionários ao público) são os mais diversos, sendo a grande maioria, animadores, destacando-se boa receptividade e interesse por parte do público e o desenvolvimento de objetos de aprendizagem para inclusão de deficientes visuais e sua imersão na aprendizagem continuada mediada por tecnologia mesmo fora da sala de aula.

Satisfazendo então a última pergunta "Quais as contribuições positivas foram viabilizadas pelas tecnologias móveis e ubíquas em sistemas para ensino/aprendizagem? ", aspectos das tecnologias móveis e ubíquas apontados pelos estudantes e professores alvos, tais como praticidade, facilidade de uso, segurança, mobilidade, interatividade, rapidez no acesso à informação e facilidade de adaptação à metodologia de ensino contribuíram significantemente para aumento de engajamento, incentivo, concentração, dinamismo, autonomia, entretenimento e enriquecimento do processo de ensino/aprendizagem.

Portanto, verifica-se que os estudos apontam contribuições positivas proporcionadas pela m-learning, evidenciando os benefícios da inserção das tecnologias móveis no processo de ensino/aprendizagem.

\section{Considerações finais e trabalhos futuros}

Nesse artigo confirma-se a existência de estudos nos últimos 10 anos relacionados às tecnologias móveis e ubíquas aplicados a educação. Percebe-se que adicionar estes dispositivos à educação significa fazer uso dos mais diversos recursos que eles disponibilizam, tornando a aprendizagem mais interativa e prazerosa na vida do aluno, com isso poderá gerar um impacto no novo modelo de aprendizado.

Os trabalhos mostram a facilidade de acesso aos dispositivos móveis e os bons resultados trazidos na inserção dos mesmos no ensino/aprendizagem, quando unidos com boas propostas pedagógicas. Há de se frisar o avanço no que diz respeito ao desenvolvimento de novas aplicações no âmbito educacional, porém estudos ainda estão sendo feitos para tornar o "m-learning" uma realidade mais presente nas escolas e na vida dos alunos.

Como trabalhos futuros, pretende-se construir um catálogo, com exemplos de diversas tecnologias móveis e metodologias possíveis para serem aplicados em escolas de ensino presencial, possibilitando aos docentes dessas escolas uma base para fomentar a inserção na sua prática pedagógica e aos alunos, possibilidade de construir uma nova forma de aprendizado por meio desses dispositivos.

\section{Referências}

Ally, M., \& Prieto-Blázquez, J. (2014). What is the future of mobile learning in education? Revista de Universidad y Sociedad del Conocimiento, 11(1), 142-151.

MORAN, José Manoel. A educação que desejamos. Novos desafios e como chegar lá. Campinas: Papirus, 2013.

Pelissoli, L., \& Loyolla, W. (2004). Aprendizado móvel (M-Learning): dispositivos e cenários. In Actas do congresso Internacional de Educação a Distância, Brasil. Recuperado de http://www.abed.org.br/congresso2004/por/htm/074-TC-C2.html Acessível em (Vol. 15, pp. 03-06) 